\title{
Students in Graduate Program Share Coping Strategies Utilized during the COVID-19 Pandemic
}

\author{
Cheryl Holden, Tesa Brown \\ Department of Masters in Healthcare Administration, University of Arkansas-Fort Smith, Fort Smith, AR, USA \\ Email: cheryl.holden@uafs.edu
}

How to cite this paper: Holden, C., \& Brown, T. (2021). Students in Graduate Program Share Coping Strategies Utilized during the COVID-19 Pandemic. Open Journal of Social Sciences, 9, 15-21. https://doi.org/10.4236/jss.2021.97002

Received: June 2, 2021

Accepted: July 6, 2021

Published: July 9, 2021

Copyright ( 2021 by author(s) and Scientific Research Publishing Inc. This work is licensed under the Creative Commons Attribution International License (CC BY 4.0).

http://creativecommons.org/licenses/by/4.0/

\begin{abstract}
While there is ample research on how the COVID-19 pandemic impacted students, data does not sufficiently address how COVID-19 affected graduate-level students enrolled in an online platform. The study completed in December 2020 aimed to answer what coping strategies students in a full online graduate program utilized during the COVID-19 pandemic. The data collection tool consisted of a questionnaire distributed via anonymous survey using university email. It is common knowledge that graduate-level students are held to higher rigor and standards. The overall perception was that graduate students should not be impacted by the pandemic's academic changes since the graduate program was entirely online pre-COVID-19. This perception proved to be untrue. The data analysis reflected mental health as the second largest area of impact for online graduate-level students due to the pandemic. In this study, graduate-level students shared coping strategies that worked well for them throughout the pandemic. This data will help fill a gap in research and hopefully spark the interest of graduate programs to offer coping strategies to promote mental health among their graduate-level students regardless of whether they are participating in traditional or online learning platforms.
\end{abstract}

\section{Keywords}

COVID-19, Graduate Students, Coping Strategies, Well-Being, Mental Health

\section{Introduction}

The spring 2020 semester started like any other semester. No one knew or had any forewarning that a pandemic would occur and change everything in a few months into the semester. COVID-19 was declared a pandemic by the World 
Health Organization (WHO) on March 11, 2020 (World Health Organization, 2020). Shortly after the WHO announcement, universities across the nation announced campus closures. The pattern for many academic institutions was to dismiss students for spring break, and when classes resumed, they would be held remotely. There was no preparation, protocols, or processes in place as most faculty and students had never experienced such a rapid succession of academic changes, much less a pandemic. Students and faculty were faced with navigating technological and logistical challenges while prioritizing the health and safety of themselves and their families. Such a rapid, unprecedented transition significantly impacted students on multiple fronts.

Before the COVID-19 pandemic, there was strong evidence reflecting thatgraduate-level students experience a high level of stress, anxiety, or depression (Arnold, 2014; Concerto et al., 2017; Stillwell et al., 2017). According to the American College of Health Association (2018), a nationally known organization for advocacy and leadership for university health, nearly $66 \%$ of graduate students experienced above average stress. Out of faculty concerns for the students in the graduate program, the research problem was created to explore what coping strategies graduate-level students utilize during COVID-19. Therefore, this study aimed to explore the coping strategies utilized by graduate students during the COVID-19 pandemic. The objectives of the study were two-fold: To better understand coping strategies utilized during the COVID-19 pandemic and identify the top coping strategies utilized by graduate-level students.

\section{Literature Review}

The COVID-19 pandemic forced classroom closures across the nation, leaving colleges and universities scrambling to convert their in-person classrooms to virtual and online learning platforms. Since this transition, researchers have begun to study how these changes have impacted students overall. The concern of negative impacts on student performance was the basis for multiple studies. Supernak et al. (2021) investigated whether the conversion to an online platform affected the coverage of student outcomes for civil engineering students. The results showed student outcomes were not significantly impacted by the COVID-19 restrictions, except for the student outcomes reliant on lab experimentation. Other researchers found similar results for students taking courses requiring lab activities (Qadir \& Al-Fuqaha, 2020). Baber (2020) found few differences between the perception of course outcomes and satisfaction among students who transitioned to an online platform. Seifert et al. (2021) found communication between students and instructors essential for success among medical students' satisfaction and positive learning outcomes after moving to an online platform. However, these studies did not address any other student issues created by the pandemic.

Another popular research theme was how to transition to a virtual or online 
instructional platform successfully. Researchers shared personal experiences on successful technological methods and best practices to ensure compassionate flexibility for students (Gelles et al., 2020). Adaptation of course assignments was also necessary. Tang et al. (2020) found that student learning and course evaluations improved when instructors used a combined online and flipped learning model. The resurgence of project-based learning also positively impacted student outcomes in the online environment (Hira \& Anderson, 2021).

Researchers also considered various socio-economic factors for students transitioning to virtual or online learning platforms. Alsandor and Trout (2020) noted how students found themselves working remotely from home in addition to their academic studies, and many were caring for school-aged children who also required online support. Asgari et al. (2021) presented several negative impacts of vulnerable student groups, particularly those who were financially disadvantaged.

It is well-known COVID-19 impacted society from a physical illness perspective and a mental health perspective. Students' mental health support was another program significantly affected by campus closures since students could no longer access face-to-face interaction with teachers and counselors. Many student support services were closed and did not offer any possibilities for virtual access (Zhai \& Du, 2020). Wasil et al. (2021) studied how the Common Elements Toolbox, adapted for students impacted by COVID-19, could be used as an online intervention for supporting students during the pandemic. Fruehwirth et al. (2021) noted an increase in anxiety and depression among students. Students indicated the main contributing factors were work reductions and social isolation. Although studies exploring coping strategies were limited, researchers noted how students used strategies such as problem solving, spirituality, social support, family connections, and counseling (Sümen \& Adıbelli, 2021; Zhao et al., 2021).

As evidenced through the literature review, researchers completed multiple studies regarding the impact of COVID-19 on students overall. This study aimed not only to consider the effects of COVID-19 for online graduate students but also which coping mechanisms they found most helpful throughout the pandemic.

\section{Methodology}

The study took place at a mid-sized university in Western Arkansas offering graduate degrees, bachelor's degrees, associate degrees, and certificate programs. Student enrollment for the university is approximately 5000 students. The target population was students in the Master of Science in Healthcare Administration (MHCA) program registered for online courses in the spring 2021 semester. The search terms were created around COVID-19 and graduate students' well-being. The results of the study identified coping strategies utilized by graduate-level students and each coping strategy was ranked by percentages to determine the top coping skills. This study was designed to increase awareness of how COVID-19 
impacted graduate-level students and identify coping strategies utilized by graduate-level students.

\subsection{Data Collection}

The data collection tool was a survey that opened with an announcement that the survey is voluntary, and respondents could opt out of the survey at any time. The survey responses were confidential and protected. Additionally, the announcement stated by clicking the "Continue" button, you are indicating that you have read and agree to participate in the survey. The survey consisted of eleven questions exploring the impact of COVID-19 on graduate-level students including impact on their educational program, their personal lives, and the coping strategies used throughout the pandemic. Each question was designed for the respondent to select one or multiple distractors and included the option "Other" for feedback.

The survey was distributed to 52 students enrolled in the graduate program via university email. Twelve respondents (23\%) completed the survey in its entirety with one respondent opting out. Although the graduate program is small and the study respondent rate low, it is a general representation of graduate-level students.

\subsection{Data Analysis and Results}

Survey data "Figure 1" was collected and analyzed, with $33.3 \%$ of respondents indicating that COVID-19 affected them mentally and $16.6 \%$ of respondents indicated they were emotionally impacted. Mentally and emotionally are often perceived by many as intertwined. The financial impact was identified as the primary area impacted at $41.67 \%$ as a result of COVID-19. This information aligns with the study's aim to explore the coping strategies utilized by graduate students during the COVID-19 pandemic.

The data analysis reflected exercise as the primary coping strategy for graduate students at $41.6 \%$, followed by social media, none and other equally at $16.67 \%$. Interestingly enough, coping strategy options for campus resources, computer, food, meditation, sleep, and television resulted in $0 \%$. Exercise being the leading coping strategy identified is compelling since most, if not all exercise facilities were closed during this time. Additionally, adhering to COVID-19 guidelines required most people to be isolated. These results offer future research opportunities such as what type of exercise, how often, and where the exercise took place.

The results of this survey have been shared with the university leadership and resulted in additional opportunities and resources for mental health to services provided to students and faculty. The telehealth counseling option was a great addition to faculty and student resources.

\subsection{Limitations}

There are a few limitations to the study. The study only collected data from one 
Q4 What coping strategies did you utilize during the pandemic?

Answered: $12 \quad$ Skipped: 0

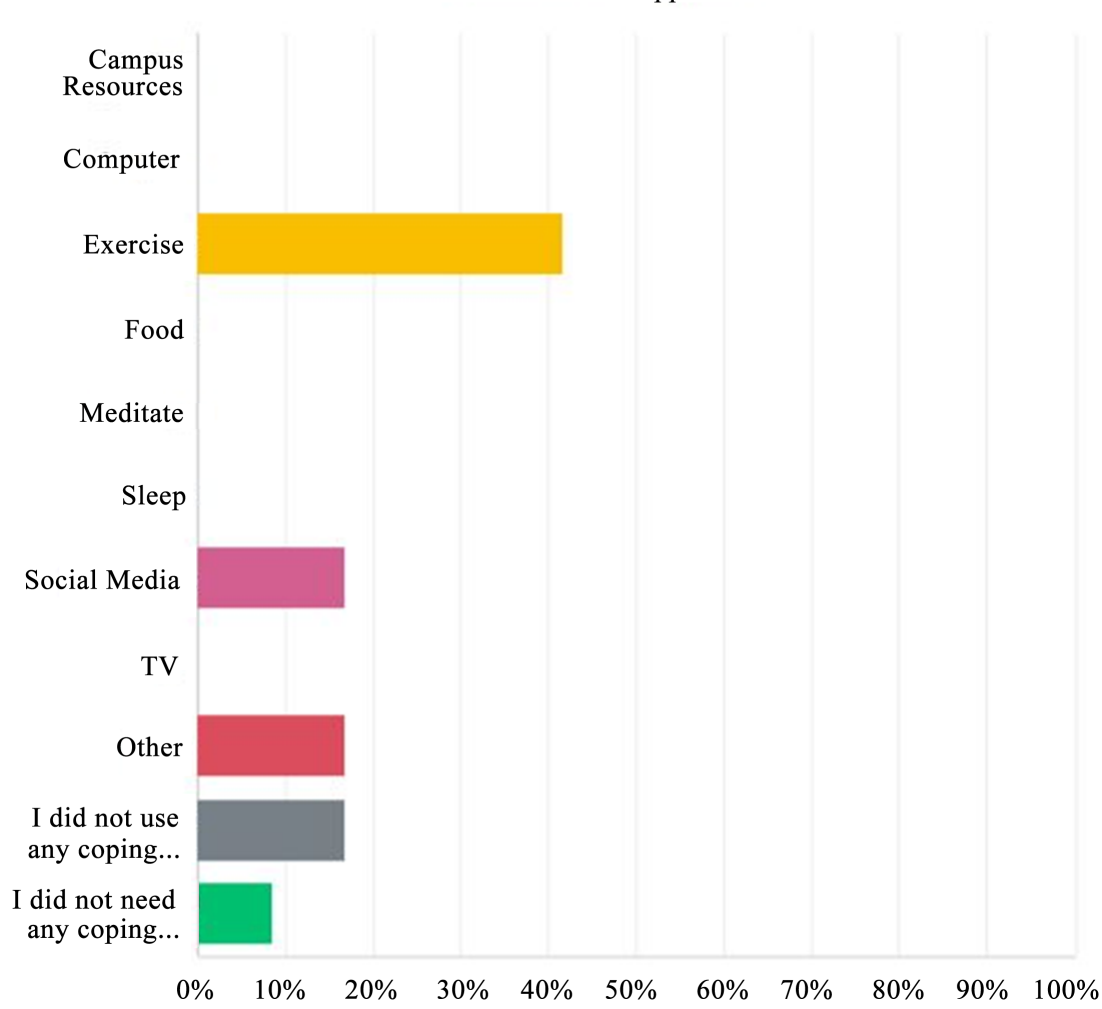

\begin{tabular}{llr} 
ANSWER CHOICES & RESPONSES & \\
Campus Resources & $0.00 \%$ & 0 \\
\hline Computer & $0.00 \%$ & 0 \\
\hline Exercise & $41.67 \%$ & 0 \\
\hline Food & $0.00 \%$ & 0 \\
\hline Meditate & $0.00 \%$ & 0 \\
\hline Sleep & $0.00 \%$ & 2 \\
\hline Social Media & $16.67 \%$ & 0 \\
\hline TV & $0.00 \%$ & 2 \\
\hline Other & $16.67 \%$ & 2 \\
\hline I did not use any coping strategies. & $16.67 \%$ & 1 \\
\hline I did not need any coping strategies. & $8.33 \%$ & 12 \\
TOTAL & &
\end{tabular}

Figure 1. Coping strategies for graduate students.

single graduate-level program, meaning one single community's experiences influenced the data. The lack of diversity could impact the accuracy of recommendations when projecting the results of this study to larger programs or populations. Second, the low number of respondents should be considered since more responses could alter the data outcomes. Timing of the survey could influence respondent rate as the survey was distributed the first week of May 2021 when for most students this can be considered finals week or wrap up week. During this period students also received several other academic surveys. 


\subsection{Ethical Considerations}

Throughout the study, the researchers considered ethical standards for research to protect all study participants. The study presented minimal risk to research participants since the study utilized a voluntary, anonymous survey to collect data. The research study received approval from the University Institutional Review Board.

\section{Conclusion}

There is a significant amount of recent research on the impact of the COVID-19 pandemic on students. However, there is limited research on the effects of the COVID-19 pandemic for online graduate students, specifically the coping strategies used by graduate students throughout the pandemic. Additionally, there is very little research and guidance on how to navigate a pandemic. This study was designed to increase the awareness of how COVID-19 impacted graduate-level students and identify coping strategies utilized by graduate-level students.

While this study will not contain all the information required to create best practice guidelines, we can incorporate student feedback to develop future programming to support graduate students regardless of their enrollment status. Leadership in academia should highly consider lessons learned by those who lived the experience and provided valuable feedback. Universities can promote mental health by assisting students with developing coping skills and provide coping resources to navigate stressful situations.

\section{Conflicts of Interest}

The authors declare no conflicts of interest regarding the publication of this paper.

\section{References}

Alsandor, D. J., \& Trout, I. Y. (2020). Graduate Student Well-Being: Learning and Living during the Covid-19 Pandemic. International Journal of Multidisciplinary Perspectives in Higher Education, 5, 150-155. https://doi.org/10.32674/jimphe.v5i1.2576

American College Health Association (2018). American College Health Association-National College Health Assessment II: Graduate and Professional Students Reference Group Executive Summary Spring 2018. Silver Spring, MD: American College Health Association.

Arnold, C. (2014, February 4). Paying Graduate Student's Mental Toll. Science Magazine. https://www.sciencemag.org/careers/2014/02/paying-graduate-schools-mental-toll

Asgari, S., Trajkovic, J., Rahmani, M., Zhang, W., Lo, R. C., \& Sciortino, A. (2021). An Observational Study of Engineering Online Education during the COVID-19 Pandemic. PLoS ONE, 16, e0250041. https://doi.org/10.1371/journal.pone.0250041

Baber, H. (2020). Determinants of Students' Perceived Learning Outcome and Satisfaction in Online Learning during the Pandemic of COVID19. Journal of Education and E-Learning Research, 7, 285-292. https://doi.org/10.20448/journal.509.2020.73.285.292

Concerto, C., Patel, D., Infortuna, C., Chusid, E., Muscatello, M. R., Bruno, A., Zoccali, 
R., Aguglia, E., \& Battaglia, F. (2017). Academic Stress Disrupts Cortical Plasticity in Graduate Students. Stress, 20, 212-216. https://doi.org/10.1080/10253890.2017.1301424

Fruehwirth, J. C., Biswas, S., \& Perreira, K. M. (2021). The Covid-19 Pandemic and Mental Health of First-Year College Students: Examining the Effect of Covid-19 Stressors Using Longitudinal Data. PLoS ONE, 16, e0247999.

https://doi.org/10.1371/journal.pone.0247999

Gelles, L. A., Lord, S. M., Hoople, G. D., Chen, D. A., \& Mejia, J. A. (2020). Compassionate Flexibility and Self-Discipline: Student Adaptation to Emergency Remote Teaching in an Integrated Engineering Energy Course during COVID-19. Education Sciences, 10, Article No. 304. https://doi.org/10.3390/educsci10110304

Hira, A., \& Anderson, E. (2021). Motivating Online Learning through Project-Based Learning during the 2020 Covid-19 Pandemic. IAFOR Journal of Education, 9, 93-110. https://doi.org/10.22492/ije.9.2.06

Qadir, J., \& Al-Fuqaha, A. (2020). A Student Primer on How to Thrive in Engineering Education during and beyond COVID-19. Education Sciences, 10, Article No. 236. https://doi.org/10.3390/educsci10090236

Seifert, T., Becker, T., Büttcher, A. F., Herwig, N., \& Raupach, T. (2021). Restructuring the Clinical Curriculum at University Medical Center Göttingen: Effects of Distance Teaching on Students' Satisfaction and Learning Outcome. GMS Journal for Medical Education, 38, Doc1. https://doi.org/10.3205/zma001397

Stillwell, S. B., Vermeesch, A. L., \& Scott, J. G. (2017). Interventions to Reduce Perceived Stress among Graduate Students: A Systematic Review with Implications for Evidence-Based Practice. Worldviews on Evidence-Based Nursing, 14, 507-513. https://doi.org/10.1111/wvn.12250

Sümen, A., \& Adıbelli, D. (2021). Nursing Students' Readiness and Coping Strategies for the Covid-19 Pandemic in Turkey. Journal of Professional Nursing, 37, 553-561. https://doi.org/10.1016/j.profnurs.2021.03.005

Supernak, J., Ramirez, A., \& Supernak, E. (2021). COVID-19: How Do Engineering Students Assess Its Impact on Their Learning? Advances in Applied Sociology, 11, 14-25. https://doi.org/10.4236/aasoci.2021.111002

Tang, T., Abuhmaid, A. M., Olaimat, M., Oudat, D. M., Aldhaeebi, M., \& Bamanger, E. (2020). Efficiency of Flipped Classroom with Online-Based Teaching under COVID-19. Interactive Learning Environments, 1-12. https://doi.org/10.1080/10494820.2020.1817761

Wasil, A. R., Taylor, M. E., Franzen, R. E., Steinberg, J. S., \& DeRubeis, R. J. (2021). Promoting Graduate Student Mental Health during COVID-19: Acceptability, Feasibility, and Perceived Utility of an Online Single-Session Intervention. Frontiers in Psychology, 11. https://doi.org/10.3389/fpsyg.2021.569785

World Health Organization (2020). WHO Characterizes COVID-19 as a Pandemic. Geneva: World Health Organization.

https://www.who.int/emergencies/diseases/novel-coronavirus-2019/events-as-they-hap pen

Zhai, Y., \& Du, X. (2020). Addressing Collegiate Mental Health amid COVID-19 Pandemic. Psychiatry Research, 288, Article ID: 113003. https://doi.org/10.1016/j.psychres.2020.113003

Zhao, S., Yin, P., Xiao, L. D., Wu, S., Li, M., Yang, X., Zhang, D., Liao, L., \& Feng, H. (2021). Nursing Home Staff Perceptions of Challenges and Coping Strategies during COVID-19 Pandemic in China. Geriatric Nursing, 42, 887-893.

https://doi.org/10.1016/j.gerinurse.2021.04.024 